\title{
NET silencing by let-7i in postural tachycardia syndrome
}

\author{
Abdul Waheed Khan, ${ }^{1,2,3}$ Mark Ziemann, ${ }^{1,2}$ Susan J. Corcoran, ${ }^{2}$ Harikrishnan K.N, ${ }^{1,2,3}$ Jun Okabe, ${ }^{1,2}$ \\ Haloom Rafehi,, ${ }^{1,2}$ Scott S. Maxwell, ${ }^{1,2}$ Murray D. Esler, ${ }^{2}$ and Assam El-Osta ${ }^{1,2,3,4}$ \\ ${ }^{1}$ Central Clinical School, Faculty of Medicine, Monash University, Victoria, Australia. ${ }^{2}$ Baker IDI Heart and Diabetes \\ Institute, The Alfred Medical Research and Education Precinct, Melbourne, Victoria, Australia. ${ }^{3}$ Department of Pathology, \\ The University of Melbourne, Parkville, Victoria, Australia. ${ }^{4}$ Hong Kong Institute of Diabetes and Obesity, Prince of Wales \\ Hospital, The Chinese University of Hong Kong, Hong Kong, China.
}

While strongly implicated in postural tachycardia syndrome (POTS), considerable controversy exists regarding norepinephrine transporter (NET) loss of function. POTS is characterized by the clinical symptoms of orthostatic intolerance, lightheadedness, tachycardia, and syncope or near syncope with upright posture. Abnormal sympathetic nervous system activity is typical, of a type which suggests dysfunction of the NET, with evidence that the gene responsible is under tight epigenetic control. Using RNA of isolated chromatin combined with massive parallel sequencing (RICh-seq) we show that let-7i miRNA suppresses NET by methyl-CpG-binding protein 2 (MeCP2). Vorinostat restores epigenetic control and NET expression in leukocytes derived from POTS participants.

Conflict of interest: The authors have declared that no conflict of interest exists.

Submitted: September 14, 2016

Accepted: February 8, 2017

Published: March 23, 2017

Reference information: JCI Insight. 2017;2(6):e90183. https:// doi.org/10.1172/jii.insight.90183.

\section{Introduction}

Postural tachycardia syndrome (POTS) is an autonomic disorder that presents with tachycardia, lightheadedness, and sometimes syncope on standing. While the etiology remains largely unknown, faulty neuronal reuptake of the sympathetic nervous system signaling neurotransmitter norepinephrine (NE) has been implicated (1). Augmented NE signaling due to impaired transmitter reuptake has been reported not only in POTS, but additionally in anxiety disorders (2), depression (3), and essential hypertension (4).

The action of NE is terminated, in part, by its uptake into presynaptic noradrenergic neurons by the plasma-membrane NE transporter (NET), which is encoded by the SLC6A2 gene, abbreviated as NET. The effectiveness of NE reuptake relies on the capacity of the NET to tightly recapture NE released by sympathetic nerves, this being approximately $90 \%$ for the heart (5). While the dramatic phenotypic effects observed in one kindred family has been located to a specific point mutation in the NET gene responsible for impaired NET function (1), extensive genotyping studies have found nominal single-nucleotide polymorphism association with POTS (6). A likely explanation is that interindividual variation in NET function is common, and in light of the clinical importance of the transporter's central role in NE regulation, rather than querying genetic variation, we investigated gene-environment interactions, asking which biological processes might be prime targets for NET gene regulation.

The first glimpse into epigenetic regulation of the NET came from ex vivo studies in depolarized neuronal cortical cells (7). Activation of NET expression in depolarized cells is tightly linked with the dissociation of the SMARCA2-methyl-CpG-binding protein 2 (MeCP2) corepressor complex (8). In a search for a molecular basis of regulation, extensive $\mathrm{CpG}$ methylation analyses using bisulfite sequencing confirm that NET gene methylation is unremarkable (7). NET activation involves hyperacetylation of histone H3 lysines 9 and $14(\mathrm{H} 3 \mathrm{~K} 9 / 14 \mathrm{ac})$ and is correlated with methylation of histone $\mathrm{H} 3$ lysine 9 (H3K9me3). Furthermore, dissociation of the $\mathrm{MeCP} 2$ corepressor complex is inversely correlated with increased histone acetylation. Subsequent human studies revealed that NET expression was indeed subject to epigenetic modification in POTS (6). While we are beginning to understand the epigenetic modifications that regulate gene expression, we still do not know the molecular machineries that target NET for reactivation. Given the biological complexity of transcriptional control, we hypothesized that the chromatin state relates to the ability of the NET promoter to perceive MeCP2 binding (9). In this context, we developed a potentially novel RNA of isolated chromatin (RICh) assay using locked nucleic acid (LNA) probes designed to target the human NET promoter. The purpose of this study was to investigate NET gene silencing with the specific aim to 
reactivate expression. We show that $\mathrm{MeCP} 2$ binding on the $N E T$ gene is mediated by the noncoding RNA (ncRNA), let-7i, and that NET expression is subject to pharmacological histone deacetylase (HDAC) inhibition, restoring specific epigenetic modifications associated with expression in POTS participants.

\section{Results}

Disambiguous capture of NET-interacting RNAs. To investigate the altered chromatin state of the NET we developed a RICh assay followed by massive parallel sequencing (RICh-seq) method to isolate interacting RNAs (Figure 1A). Three specific LNA probes were designed against the human NET promoter aided by the ENCODE project dataset including transcription factor binding sites, chromatin accessibility, and active histone marks (Supplemental Figure 1; supplemental material available online with this article; https://doi. org/10.1172/jci.insight.90183DS1). LNA probes were designed to capture an almost 1.2-kbp region of the human NET promoter (Figure 1B). In experiments used to optimize assay conditions, single-strand LNA probes were assessed for soluble-chromatin pulldown (Figure 1C). Scrambled-sequence probes were used to assess the specificity of LNA-chromatin hybrids. NET-associated RNAs were captured using streptavidin beads and purified RNA was used in the library constructions for massive parallel sequencing. Detection of the NET promoter using quantitative PCR (qPCR) from RICh-captured DNA shows the specificity of the LNA probes (Figure 1, D-F) as well as regional specificity of the probe against exon 5 of the NET gene (Figure 1G). RICh-seq generated 1.2 million reads for the specific NET probes, 2.5 million reads for input control, and 0.5 million for the scrambled control (Supplemental Table 1). RNA species enriched by the NET probes were determined for 2 control groups: (a) total chromatin RNAs, and (b) scrambled-sequence probe-bound RNAs. We identified 18 RNAs at the NET promoter compared with the input control, and 9 enriched RNAs when compared with the scrambled-probe control (Figure 2A). RNA species interacting with the NET promoter include protein-coding and ncRNAs such as miRNA, small nucleolar RNA (snoRNA), and small nuclear RNA (snRNA) (Figure 2B). K-means clustering of RNAs associated with the NET promoter identified 6 strongly enriched genes (see cluster 2, Figure 2C). Of the 6, five RNAs (MIRLET7I [let-7i], SCARNA4, SNORA46, SNORD96, and TUFM) were significantly enriched using the NET probe in both groups (FDR $<0.05$, as shown in cluster 2 of Figure 2C and summarized in Supplemental Table 2). Taken together, these results suggest that the RICh technique using LNA probes can selectively capture chromatinized NET-associated RNAs.

Chromatinization of NET involves MeCP2 and let-7i interactions in POTS. Having identified NET-interacting transcripts, we investigated the expression of specific RNAs in a case and control cohort. The case group included 12 participants with POTS (9 females, 3 males) that underwent exhaustive medical evaluation to exclude any other relevant medical condition. They share the common clinical characteristics central to the diagnosis: recurrent episodes of presyncope while standing, freedom from postural hypotension despite the presence of postural dizziness, and the presence of posture-related tachycardia (10). The healthy reference group had none of these symptoms in the 12 participants ( 9 females, 3 males). The characteristics of these 24 participants are summarized in Table 1.

Since the NET gene has been implicated in the pathophysiology of participants matched with POTS, we assessed expression in leukocytes compared with other cell types. Interestingly, NET gene expression was inversely regulated with let-7i RNA expression (Supplemental Figure 2). Of the 5 RNA transcripts detected by RICh-seq, let-7i was significantly increased $(P<0.01)$ in the POTS group (Figure 3A). RNA hybrid prediction identified 3 let-7i consensus binding sites, which were validated by RICh at the NET promoter (Supplemental Figure 3). To exclude the possibility that the let-7i interaction is a result of nonspecific binding, we assessed LNA probes with scrambled sequences using the RICh protocol. We observed no change in let-7i binding at the NET promoter using scrambled LNA probes. Chromatin from the POTS group was significantly enriched for let-7i $(P<0.01)$, consistent with binding at the NET promoter (Figure $3 \mathrm{~B})$. These experimental results indicate that in leukocytes the NET promoter is subject to let-7i-mediated chromatin interaction.

Because MeCP2 is implicated in NET silencing but of unknown binding function (6), we next assessed physical interaction with let-7i. Soluble chromatin fractions were prepared from healthy and POTS participants and RNA immunopurifications (RIPs) were performed with an antibody that specifically recognizes the MeCP2 epitope. To exclude the possibility of indirect MeCP2 binding, RIPs were performed with IgG control antibody. Chromatin derived from POTS participants was significantly enriched for let-7i with MeCP2 $(P<0.01$, Figure 3C). Furthermore, ChIP experiments confirm that $\mathrm{MeCP} 2$ interacts with the NET promoter at the same sites the LNA probes were designed to capture 


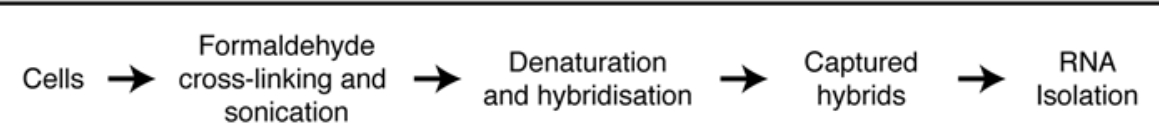

B NET gene on Chromosome 16 (q12.2): $55689225-55691050$

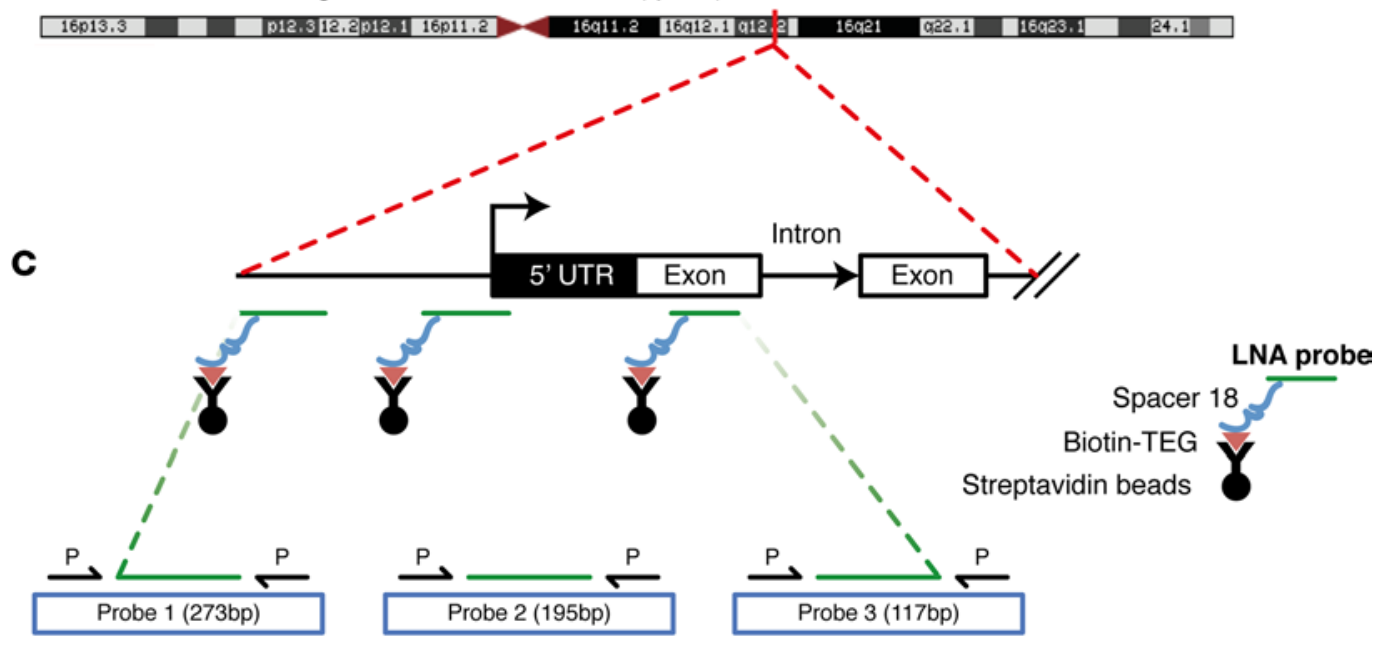

D

NET promoter DNA (Probe 1)

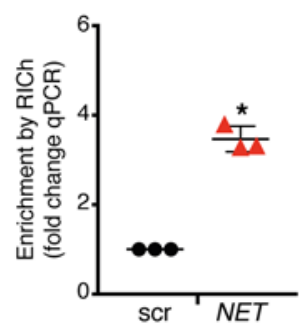

$\mathbf{E}$

NET promoter DNA (Probe 2)

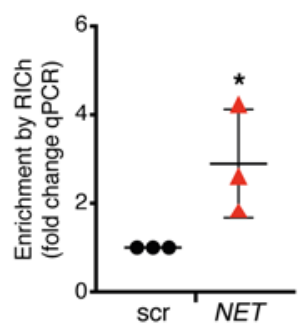

$\mathbf{F}$

NET promoter DNA (Probe 3)

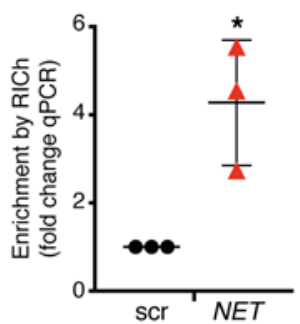

G

NET exon 5 DNA

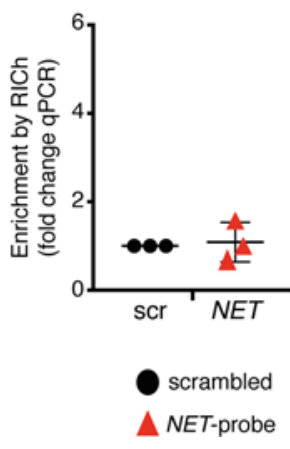

Figure 1. Optimization of RNA of isolated chromatin (RICh) assay. (A) RICh protocol. Briefly, cells (HeLa or leukocytes) were crosslinked with $1 \%$ formaldehyde and soluble chromatin prepared by sonication. Chromatin was denatured and hybridized with locked nucleic acid (LNA) probes. Chromatin-LNA probe hybrids were captured with streptavidin beads. For RICh optimization, DNA was isolated and assessed for norepinephrine transporter (NET) promoter amplification by quantitative PCR (qPCR). (B) Location of sequence-specific LNA probes targeting the NET promoter. (C) NET promoter regions are represented and correspond to those captured using sequence-specific LNA probes. Primers in schematic are abbreviated as P. (D-F) NET promoter DNA amplification by qPCR using RICh-captured DNA. (C) Amplification of exon 5 of the NET gene from RICh-captured DNA shows no difference between the NET-specific and scrambled (scr) probes. This is a measure of assay specificity; $n=3$, mean \pm SD. ${ }^{*} P<0.05$ by Student's $t$ test.

(Supplemental Figure 4, A-C). Taken together, these results show increased MeCP2 and let-7i chromatin interaction in leukocytes derived from POTS participants.

RNA-dependent MeCP2 binding on NET. Having demonstrated that MeCP2 and let-7i are associated with $N E T$, we investigated the effect of MeCP2 loss on NET-mediated transcription using lentivirus shRNA. Because lentiviral transduction of primary untransformed leukocytes is notoriously difficult (11), we performed MeCP2 knockdown (KD) using nonprimary immortalized cells (Figure 4A). NET expression was significantly increased in MeCP2 KD cells $(P<0.01$ Figure 4B). Since MeCP2 interacts with chromatin 
A
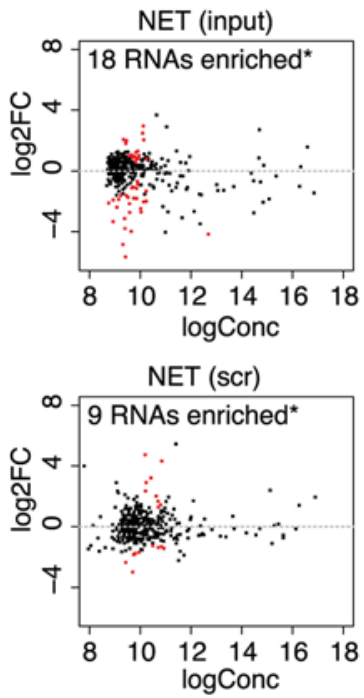

B

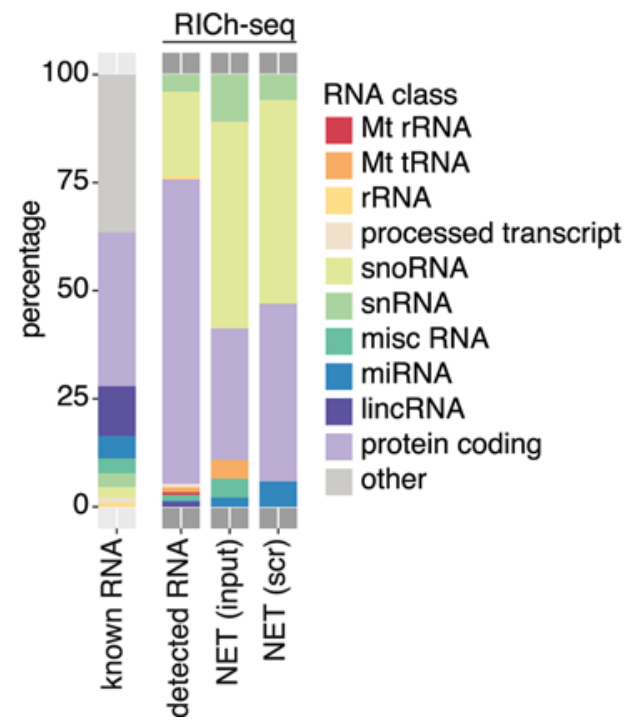

C

RNAs associated with NET gene

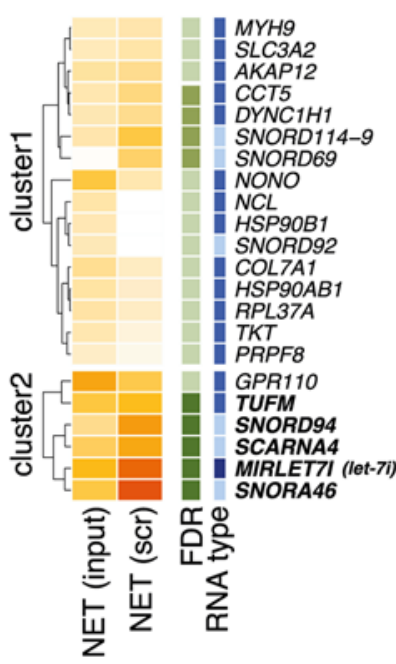

$\log \mathrm{FC}$

4

3

2

1

0

RNA type

snoRNA

protein coding miRNA

FDR $p$ value $<0.05$

NET (input) only

NET (scr) only

both

Figure 2. RICh-seq identifies NET promoter-specific RNAs. RNA of isolated chromatin (RICh) combined with massive parallel sequencing (RICh-seq) was undertaken to identify norepinephrine transporter (NET) promoter-specific RNAs. (A) MA plots of RNA detected at the NET promoter compared with input RNA (total chromatin-bound RNA) and scrambled-probe controls. ${ }^{*}$ Red data points indicate FDR $<0.05$. (B) A stacked bar chart showing RNA classes detected by RICh-seq in the input and scrambled datasets. (C) A heatmap showing k-means clustering based on the log(fold change) (logFC) of RNA transcripts enriched at the NET promoter with an FDR $<0.05$ in at least 1 comparison. RNAs with FDR $<0.05$ in both comparisons are highlighted in bold; $n=3$. Mt, mitochondrial; snoRNA, small nucleolar RNA; snRNA, small nuclear RNA; misc RNA, miscellaneous RNA; scr, scramble; lincRNA, long intergenic noncoding RNA.

and also RNA-dependent protein complexes with high affinity (12), we hypothesized that $N E T$ silencing by $\mathrm{MeCP} 2$ is mediated by chromatin interaction with let-7i. To assess the specificity of MeCP2 binding to the NET promoter, ChIP assays were performed with MeCP2 antibody and association with the NET promoter assessed by qPCR. As shown in Figure 4C, chromatin was significantly enriched for MeCP2. To address the possibility that recruitment of $\mathrm{MeCP} 2$ on $N E T$ is subject to RNA-mediated interactions we devised a ChIP-RNase technique (Figure 4D). In this assay, soluble MeCP2-associated chromatin was subject to endoribonuclease A (RNase A), which catalyzes the cleavage of single-strand RNA molecules. Soluble chromatin not exposed to RNase A degradation was significantly enriched for MeCP2 on the NET promoter when compared with IgG antibody controls. Furthermore, MeCP2 binding at the NET promoter was significantly reduced in RNase-treated chromatin (Figure 4E). The ChIP-RNAse results support the notion that MeCP2 localization on NET chromatin is dependent on RNA. To confirm that the loss of MeCP2 binding following RNAse was specific for the NET promoter, we assessed known MeCP2 target genes such as IL-6 (ILG), which is suppressed by MeCP2 binding (13). In ChIP assays we recovered IL6 DNA equally well from -RNAse and +RNAse chromatin isolates using MeCP2 antibody (Figure 4F). These results suggest that $N E T$ is subject to RNA-dependent MeCP2 interactions.

Vorinostat restores let-7i-mediated NET silencing. MeCP2 resides in a co-repressor complex with HDAC activity (14), and vorinostat (suberoylanilide hydroxamic acid or SAHA) has been approved by the FDA for the treatment of hematological malignancies and is currently being evaluated for other diseases (15). Using human leukocytes isolated from healthy and POTS participants, we developed an experimental assay to test $N E T$ reactivation because there are no comparative ex vivo models or human studies to assess the efficacy of therapies directly in POTS. Leukocytes stimulated with the paradigmatic HDAC inhibitor trichostatin A (TSA) show increased NET expression when assessed by quantitative reverse transcription PCR (qRT-PCR) (Supplemental Figure 5, A-C). Next, we investigated whether ex vivo treatment using single-dose exposure to $2 \mu \mathrm{M}$ vorinostat could reactivate $N E T$ expression. Treatment of leukocytes derived from POTS participants with vorinostat caused a dramatic upregulation of $N E T$ expression (Figure $5 \mathrm{~A}$ ). To exclude the possibility that increased NET expression occurs as a result of ex vivo maintenance of human leukocytes in primary conditions, we examined genes that are relevant to leukocyte response and regulated by vorinostat. TNFSF9 is a member of the TNF (ligand) superfamily and is induced by pharmacological HDAC inhibition (16). TP53 
Table 1. Demographic data of healthy and postural tachycardia syndrome (POTS) groups, and head-up tilt (HUT)A results for the POTS cohort

\begin{tabular}{|c|c|c|c|}
\hline & Controls & POTS & $\boldsymbol{P}$ \\
\hline Sex, F/M & $9 / 3$ & $9 / 3$ & \\
\hline Age, y & $32 \pm 3$ & $32 \pm 3$ & 0.84 \\
\hline Height, m & $1.72 \pm 0.06$ & $1.72 \pm 0.04$ & 0.93 \\
\hline Weight, kg & $62 \pm 3$ & $81 \pm 7$ & 0.001 \\
\hline BMI & $21 \pm 1$ & $26 \pm 2$ & 0.006 \\
\hline Supine SBP, mmHg & & $127 \pm 3$ & \\
\hline Supine DBP, mmHg & & $67 \pm 2$ & \\
\hline Supine HR, bpm & & $85 \pm 4$ & \\
\hline End HUT SBP, mmHg & & $132 \pm 7$ & \\
\hline End HUT DBP, mmHg & & $73 \pm 4$ & \\
\hline End HUT HR, bpm & & $124 \pm 4$ & \\
\hline$\Delta \mathrm{HR}, \mathrm{bpm}$ & & $39 \pm 5$ & \\
\hline Supine Total NE spillover, ng/min & & $627 \pm 179$ & \\
\hline Supine MSNA, bursts per $\min (n=7)$ & & $21 \pm 4$ & \\
\hline End HUT Total NE spillover, ng/min & & $801 \pm 79$ & \\
\hline End HUT MSNA, bursts per $\min (n=4)$ & & $35 \pm 6$ & \\
\hline \multicolumn{4}{|c|}{$\begin{array}{l}\text { AThe healthy reference population did not undergo HUT testing. } P \text { values determined b } \\
\text { Student's } t \text { test. SBP, systolic blood pressure; DBP, diastolic blood pressure; HR, heart } \\
\text { rate; NE, norepinephrine; MSNA, muscle sympathetic nerve activity; bpm, beats per } \\
\text { minute; F, female; M, male; } y \text {, years. All results expressed as mean } \pm \text { SEM. }\end{array}$} \\
\hline
\end{tabular}

is a protein-coding gene that acts as a tumor suppressor and is suppressed by vorinostat treatment (17). The expression of TNFSF9 increased (Figure 5B) and TP53 decreased (Figure 5C) in cells stimulated by vorinostat. Taken together, the results of our preclinical investigation of an epigenetic mechanism indicate NET reactivation using pharmacological HDAC inhibitors in leukocytes derived from POTS participants.

\section{Discussion}

It is probable that the faulty neuronal reuptake of the sympathetic transmitter we describe is responsible for key clinical manifestations of POTS. The diagnostic supranormal increase in heart rate with standing most probably derives from augmentation of the reflex increase in the sympathetic outflow to the heart with standing. The disposition of NE after release from sympathetic nerves is more dependent on neuronal reuptake in the heart than in all other organs. Persistence of NE in the synaptic space of the cardiac sympathetic nerves, and inappropriate tachycardia, would be a consequence of a neuronal NE reuptake defect.

POTS is characterized by postural syncope, but surprisingly without postural hypotension. The mechanism of the syncope is a pronounced reflex fall in cere-

bral blood flow on standing (18). As for the postural tachycardia, the probable cause is augmentation of a reflex sympathetic response to posture, this time in the cerebral blood vessels, by impaired neuronal reuptake of NE in POTS patients. Despite previous disagreement on this point, the human cerebral blood vessels have been demonstrated to have a functional sympathetic innervation (19). The cerebrovascular sympathetic nerves in healthy subjects have demonstrable capacity for NE reuptake, and are subject to CNS regulatory control.

Recent reports suggest that ncRNA interactions can induce significant structural changes to chromatin and regulate gene transcription (20). Demonstrable evidence indicates that $\mathrm{MeCP} 2$ coexists with regulatory determinants that recruit HDAC activity and is thought to be functionally important in gene silencing (21). The experimental results presented here suggest that NET silencing involves an HDAC complex that is subject to pharmacological inhibition $(7,22)$. Indeed, this is consistent with a model in which NET chromatinization is probably mediated by MeCP2 (6). In this study, we did not observe demonstrable epigenetic changes influenced by age or gender in POTS and healthy participants.

The findings presented are potentially novel for 2 reasons. First, to our knowledge there are no reports of $\mathrm{MeCP} 2$ binding at unmethylated loci altering gene expression in human disease. It is commonly assumed that the dominant role of $\mathrm{MeCP} 2$ as a silencing determinant at $\mathrm{CpG}$ sites is dependent on methylation (23). While recent studies have shown that the binding preferences of MeCP2 are broader than previously described (24) and can be associated with transcriptional activation (9), no studies have implicated gene silencing with let-7i. Whereas $\mathrm{MeCP} 2$ has been associated with silencing at unmethylated genes, the molecular connection to form that inactive chromatin conformation has been missing $(25,26)$. Second, and most relevant clinically, to our knowledge our study is the first to demonstrate that pharmacological HDAC inhibition can partly attenuate let-7i-mediated gene silencing by MeCP2 to reactivate NET expression.

The combination of agents often used to manage symptoms may not necessarily lend itself to accurate interpretation and influence of distinct epigenetic properties underlying chromatin modifications subject to let-7i regulation. While propranolol has been proposed to alter the expression of several miRNAs, this proposition is largely supported in small animal studies (27) and our preclinical results based on the cohort size do not support the pharmacological function of propranolol as a regulator of let-7i. Neither propranolol nor atenolol predicted let-7i expression and such observations should be tested independently. While a combination of pharmacological agents is often used to treat the symptoms of POTS, which may offer yet 
A

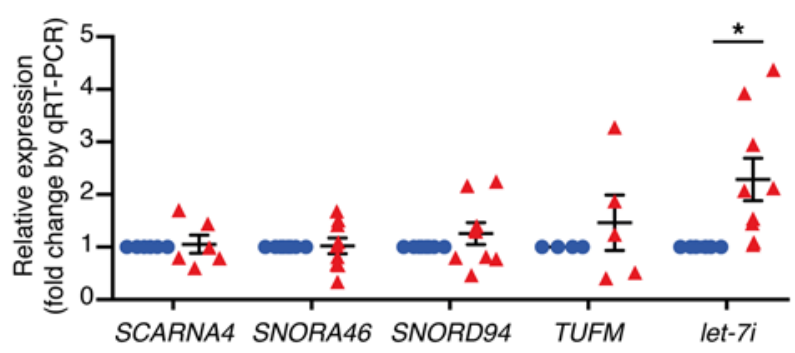

B NET bound let-7i in leukocytes

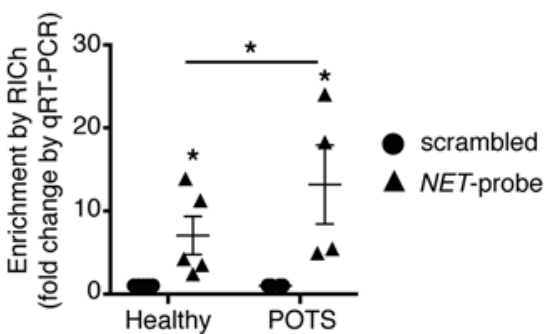

C MeCP2 bound let-7i in leukocytes

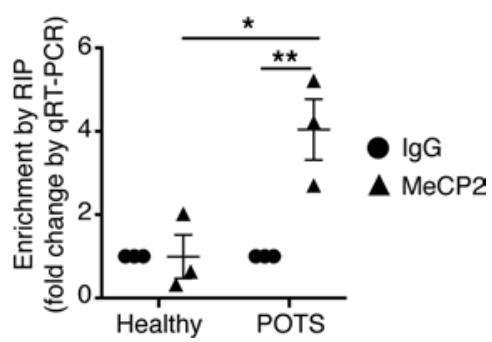

Figure 3. Chromatinization of NET involves MeCP2 and let-7i interactions in POTS leukocytes. (A) Expression of norepinephrine transporter (NET) promoter-bound RNAs SCARNA4, SNORA46, SNORD94, TUFM, and let-7i in leukocytes isolated from postural tachycardia syndrome (POTS) and healthy participants assessed by quantitative reverse transcription PCR (qRT-PCR) $(n=9)$. Relative expression was measured against U6-2 using qRT-PCR. (B) Let-7i interacts with NET chromatin. RNA of isolated chromatin (RICh) assay was performed in leukocytes derived from healthy $(n=5)$ and POTS $(n=4)$ participants and shows let-7i binding at the NET promoter using qRT-PCR. (C) MethylCpG-binding protein 2 (MeCP2) interacts with let-7i. RNA immunopurification (RIP) assay using antiMeCP2 antibody followed by the detection of let-7i using qRT-PCR $(n=3)$. Data are the mean \pm SEM. ${ }^{*} P$ $\leq 0.05,{ }^{* *} P \leq 0.01$ by Student's $t$ test.

unforeseen synergistic benefit, further studies are warranted to better understand the underlying drug interactions that confer transcriptional control of NET. With the identification of miR-

NAs such as let-7i that participate in regulatory control with MeCP2, the challenge is now to understand their biological function and signaling interactions with specific pharmacological therapies. This remains an important area of clinical-epigenetic investigation.

MeCP2 can bind double-stranded RNA molecules (28) and a protein interaction screen has identified an RNA-dependent class of spliceosome determinants (29). The screen also identified MeCP2-associated RNA including MALAT1, a long ncRNA implicated in regulating blood vessel growth $(30,31)$, as well as RNCR3, which is thought to be atheroprotective (32). MeCP2 coexists in distinct complexes that support a functional role in RNA processing and transcriptional suppression (33). This lends further support to the concept that one possible role for let-7i interaction with chromatin is to lock NET into a silent chromatin state. Consistent with our findings, vorinostat alone does not fully reactivate silent alleles, which is in contrast to promoters that are open to histone acetylation at consensus binding sites (34). Indeed, in that same study a survey of human loci by massive parallel sequencing indicated that vorinostat significantly altered the expression of genes that code for ncRNAs. It is probable that chromatinization by MeCP2 and let-7i synergistically suppress NET expression. Evidence for a functional relationship linking histone modifications with NET gene reactivation has come from studies showing that depolarization of neuronal cortical cells can stimulate increased acetylation of histone $\mathrm{H} 3$ lysines 9 and 14 (H3K9/14ac) while reducing methylation of histone $\mathrm{H} 3$ lysine 9 (H3K9me3) (7). Our results imply that acetylated histones are associated with the NET in healthy but not POTS cells. Inhibition of HDACs by TSA and vorinostat increases the acetylation level of $\mathrm{H} 3 \mathrm{~K} 9 / 14$ and activates gene transcription (34). Several lines of experimental evidence show protective benefits of HDAC inhibitors such as TSA in preclinical models of cardiac hypertrophy and ischemia $(35,36)$. Vorinostat has shown preclinical translation in small- and large-animal models $(37,38)$. While the molecular mechanisms underlying vorinostat's cardioprotective effects remain unknown, recent evidence shows that widespread acetylation and predominant deacetylation of gene targets participate in core regulatory pathways (34). Some of the connections between signaling networks and pharmacological HDAC inhibition are starting to be revealed at the molecular level (39). For example, broad histone deacetylation conferred by TSA and vorinostat are mediated by the loss of EP300/CREBBP binding at multiple gene targets. Furthermore, vascular deacetylation by vorinostat and TSA mediates widespread changes in the expression of ncRNAs and suggests crucial roles in chromatin structure and function (40).

While other transcriptional factors need to be considered with respect to regulation, our data suggest a 3-way connection with chromatin mediated by HDAC activity accompanied by let-7i and MeCP2 interaction on the NET gene. In this context, we posit that let-7i silencing of $N E T$ by MeCP2 is amenable to consequent inhibition of HDAC activity. To maximally achieve gene reactivation, it may be necessary to simultaneously block the events that are essential to the formation and maintenance of silent NET chromatin. 
A

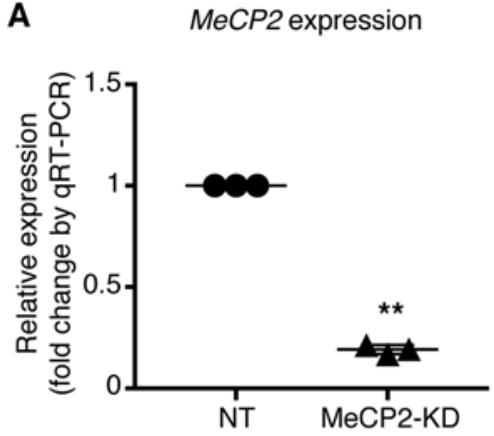

B

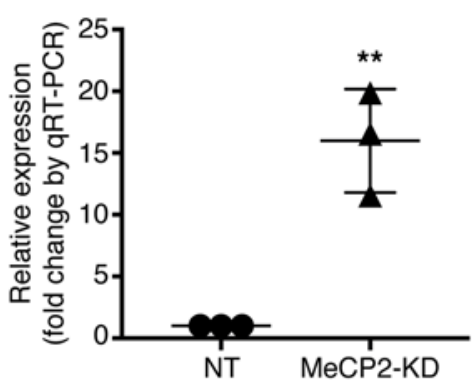

C

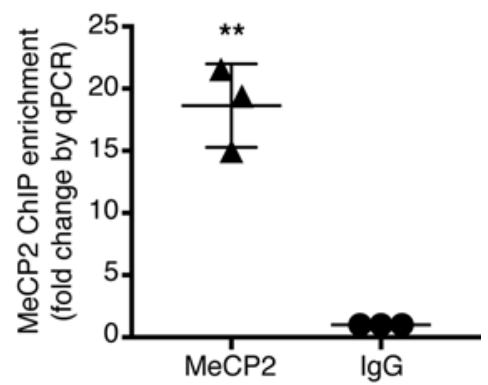

D ChIP-RNAse protocol
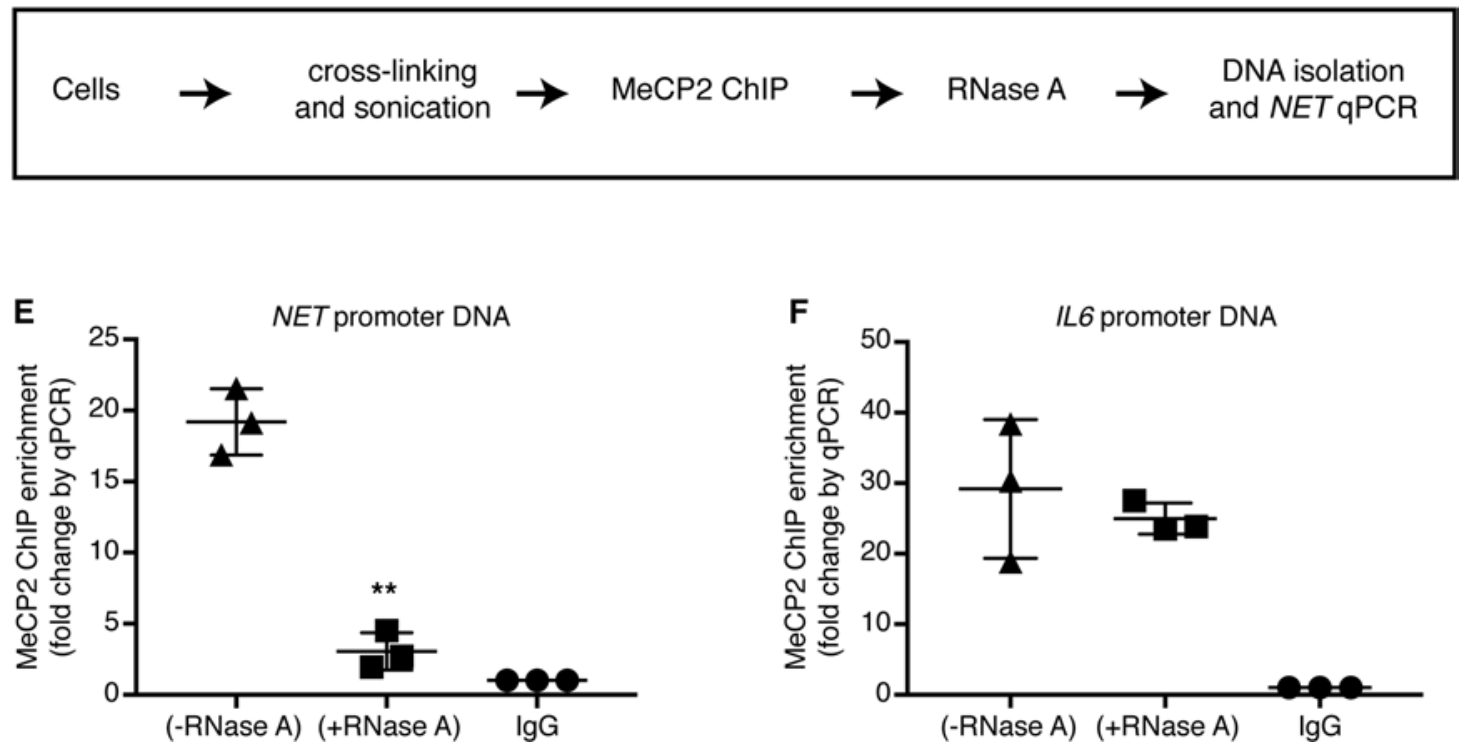

Figure 4. RNA-dependent MeCP2 binding at the NET promoter. (A) Methyl-CpG-binding protein 2 (MECP2) shRNA knockdown in HeLa cells $(n=3)$. Relative expression was measured against ACTB using quantitative reverse transcription PCR (qRT-PCR). (B) Norepinephrine transporter (NET) expression in MECP2-depleted cells $(n=3)$. Relative expression was measured against ACTB using qRT-PCR. (C) ChIP assay using an anti-MeCP2 antibody indicates binding at the NET promoter relative to IgG antibody $(n=3)$. (D) Schema of MeCP2 ChIP protocol followed by RNase treatment (ChIP-RNase) and detection of NET by qPCR. Briefly, HeLa cells were crosslinked ( $1 \%$ formaldehyde) and soluble chromatin prepared by sonication. Anti-MeCP2 antibody was used to perform ChIP. Immunopurified complexes were treated with RNase A and unbound chromatin removed by washing. Remaining DNA was purified and NET detected using qPCR. (E) ChIP-RNase assay shows that MeCP2 binding on the NET promoter is RNA dependent $(n=3)$. (F) ChIP-RNase assay shows that MeCP2 binding at the IL6 promoter is not RNA dependent $(n=3)$. Data are the mean \pm SD. ${ }^{* *} P \leq 0.01$ by Student's $t$ test.

Taken together, our results indicate that pharmacological HDAC inhibition by vorinostat may represent a therapeutic strategy that merits ongoing effort to develop novel chromatin-based treatments for POTS. Furthermore, because we observe an impaired neuronal NE reuptake phenotype in essential hypertension, panic disorder, and depressive illness, clinical epigenomic studies in essential hypertension have commenced.

\section{Methods}

RICh-seq and LNA probe design. The NET promoter region was selected by analyzing the ENCODE data for chromatin accessibility, binding sites of transcription factors, and active histone marks (41) as illustrated in Supplemental Figure 1. Three probes were designed at Chr16 (q12.2): 55689225-55691050 (GRCh37/hg19) using Stellaris probe designer version 4.1. Replacing almost 50\% of DNA nucleotides with LNA residues increased probe-chromatin interaction stability. These DNA-LNA probes were modified at their $5^{\prime}$ end by a Biotin-TEG-iSP18 spacer using LNA Oligo design tools; these probes were manufactured by Exiqon. A mixture of equal amounts of all 3 probes was used to capture the chromatin of interest. To verify that the $N E T$ promoter was captured specifically by $N E T$ probes, a probe with a scrambled sequence at equal concentration was included. 
A

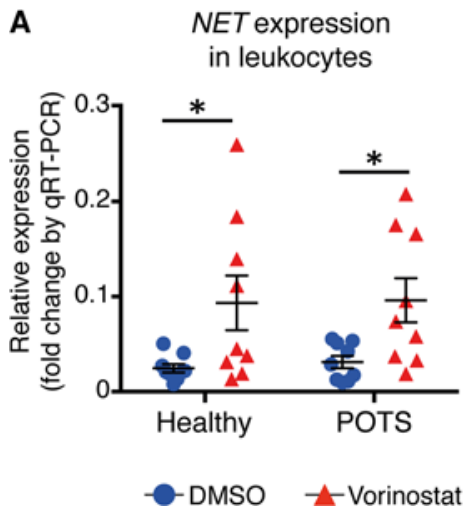

B

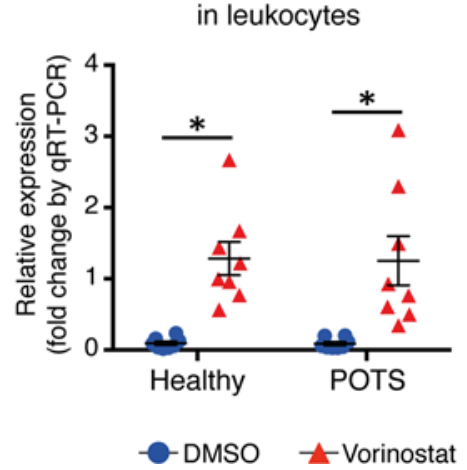

C

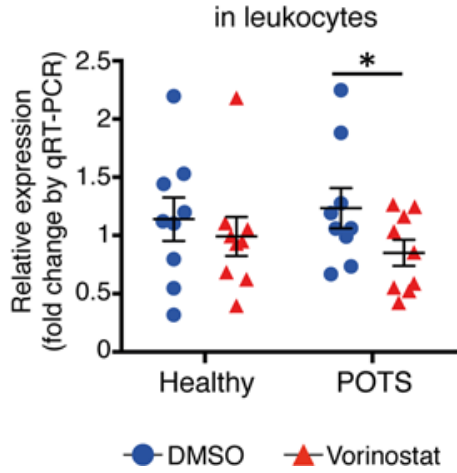

Figure 5. Vorinostat reactivates NET expression in POTS-derived leukocytes. (A) Norepinephrine transporter (NET) expression following vorinostat stimulation of leukocytes derived from healthy and postural tachycardia syndrome (POTS) participants $(n=9)$. Relative expression was measured against ACTB using quantitative reverse transcription PCR (qRT-PCR). (B) TNFSF9 expression following vorinostat stimulation of leukocytes derived from healthy and POTS participants $(n=9)$. Relative expression was measured against ACTB using qRT-PCR. (C) TP53 expression following vorinostat stimulation of leukocytes derived from healthy and POTS participants $(n=9)$. Relative expression was measured against ACTB using qRT-PCR. Data are the mean \pm SEM. ${ }^{*} P \leq 0.05$ by Student's $t$ test.

Probe sequences are as follows (+ represents LNA nucleotide): NET-1, 5'-A+GT+GA+CA+GACT+C TC+CAGAAC-3'; NET-2, 5'-T+GCA+CTA+CAAA+CTC+GCTA+GC-3'; NET-3, 5'-A+CA+GCA+G GAA+GT+CGAT+CTTC-3'; Scrambled, 5'-GC+GA+CA+CC+GCAA+CTA+GATTA-3'.

Soluble chromatin preparation. HeLa cells $\left(3 \times 10^{7}\right)$ were crosslinked with $1 \%$ formaldehyde for $10 \mathrm{~min}$ utes at room temperature. Collected cell pellets were lysed in lysis buffer (1\% SDS, $10 \mathrm{mM}$ EDTA, and 50 $\mathrm{mM}$ Tris- $\mathrm{HCl} \mathrm{pH}$ 8.0). Cell lysates were sonicated using a Bioruptor (Diagenode) with constant power settings. Soluble chromatin was 5-fold diluted in chromatin dilution buffer $(0.01 \%$ SDS, $1.1 \%$ Triton X-100, 1.2 mM EDTA, 1 mM EGTA, 167 mM NaCl, 16.7 mM Tris- $\mathrm{HCl} \mathrm{pH}$ 8.0).

Chromatin capture. M-270 streptavidin Dynabeads were washed and blocked with salmon sperm DNA in binding buffer (10 mM Tris pH 7.5, $1 \mathrm{mM}$ EDTA, $1 \mathrm{M} \mathrm{NaCl}, 0.1 \%$ Tween 20). NET probes and scrambled probes $(100 \mathrm{pmol})$ were added to $3 \mu \mathrm{g}$ of diluted crosslinked chromatin and incubated at $95^{\circ} \mathrm{C}$ for 5 minutes, $37^{\circ} \mathrm{C}$ for 30 minutes, $72^{\circ} \mathrm{C}$ for 2 minutes, $37^{\circ} \mathrm{C}$ for 30 minutes, $72{ }^{\circ} \mathrm{C}$ for $2 \mathrm{~min}-$ utes, and $37^{\circ} \mathrm{C}$ for 30 minutes using a thermal cycler. Multiple reactions were performed and the captured chromatin was pooled. Hybridized biotin probe/chromatin isolates were captured with blocked streptavidin Dynabeads and reverse crosslinked with proteinase $\mathrm{K}$ at $62^{\circ} \mathrm{C}$ for 45 minutes before RNA purification. The RNAs associated with captured chromatin and input (total chromatin-bound RNA) underwent small RNA-seq library preparation with the NEBNext Small RNA Library Prep Set for Illumina and sequenced with Illumina MiSeq sequencing platform for 36 cycles with version 2 reagents. Validation of let-7i and NET promoter interaction was performed using qRT-PCR and miScript II RT kits from Qiagen.

Bioinformatic analysis. Short reads underwent quality trimming using a quality threshold of 10 and minimum length of 18 nucleotides followed by clipping the adapter sequence with Fastx- Toolkit. Filtered reads were mapped to the human genome using STAR aligner (42). Human genome sequence and version 78 annotations were obtained from the Ensembl FTP site (43). FeatureCounts was used to count reads mapped to exons and generate the expression count matrix (44). Differential analysis was undertaken using edgeR correcting for batch variation (45). A Benjamini-Hochberg FDR-adjusted $P$ value of less than or equal to 0.05 was considered significant. Two comparisons were undertaken: (a) NET-probe-bound versus scrambled-probe-bound and (b) NET-probe-bound versus total input RNA. The intersection of these 2 gene lists was considered candidate binders to the NET promoter. The RICh-seq results were deposited in the NCBI's Gene Expression Omnibus (GEO GSE93235).

RIP. Soluble chromatin was prepared using micrococcal nuclease enzyme in human leukocytes. To ensure RNA integrity, only freshly prepared buffers containing RNase inhibitors were used. An antibody that recognizes the MeCP2 epitope (Sigma-Aldrich, 9317) was used in immunoprecipitation $\left(4^{\circ} \mathrm{C}\right.$ for 4 hours) to purify RNA associated with MeCP2. RNA was purified from conjugates using a Qiagen RNeasy kit. qRT-PCR and miScript II RT were used for let-7i and MeCP2 interactions. 
shRNA-mediated KD strategies. The KD of MeCP2 protein in HeLa cells was achieved by MISSION shRNA-expressing lentiviral vectors (Sigma-Aldrich) specifically targeting human MeCP2 (RefSeq sequence NM_001110792). The target sequence for MeCP2 was 5'-CGTCTGCAAAGAGGAGAAGAT-3'. MeCP2 $\mathrm{KD}$ in the cells was verified by qRT-PCR with MECP2-specific primers. Cells transduced with MISSION nontarget shRNA vector served as controls.

RNase treatment of immunopurified chromatin (ChIP-RNase). Soluble chromatin was prepared with $1 \%$ formaldehyde and sonication using the Bioruptor. Immunoprecipitation was performed by incubating crosslinked chromatin with anti-MeCP2 antibody at $4^{\circ} \mathrm{C}$ for 4 hours. The immunopurified chromatin was subject to RNase A treatment ( $20 \mu \mathrm{g})$ in $100 \mu \mathrm{l}$ of $1 \mathrm{M} \mathrm{NaCl}$ in Tris-EDTA (TE). Input and antibody-bound isolates were heated at $62^{\circ} \mathrm{C}$ for 45 minutes in elution buffer containing proteinase $\mathrm{K}$ and reversed crosslinked DNA was purified with a NucleoSpin Extract II Kit (Macherey-Nagel). ChIP enriched DNA sequences ( \pm RNase A) were detected by amplification with qRT-PCR using NET-specific primers. Percentage input was calculated and results are presented as relative fold change for the target sequence compared with IgG control.

Primers used in RICh, ChIP-RNase, MeCP-KD, and RIP experiments were: NET (region 1) forward GTGCTGGGCTGTGACCTGTCA and reverse GGGAATGTGCGGCTCAGGGC; NET (region 2) forward GCCCTAGAGCCTGCCAAG and reverse GCCTTCTCGGACACTTAACG; NET (region 3) forward AGCTGCTGGTGGTGAAGG and reverse CGACTACGGACAGCAGGAAG; NET (exon 5) forward AGGAAGGTGGGAAAGAGCAT and reverse GCACGAACAGCACGAAGTAA; IL6 forward TGCACTTTTCCCCCTAGTTG and reverse TCATGGGAAAATCCCACATT; MECP2 mRNA forward ACTCCCCAGAATACACCTTGCTT and reverse TGAGGCCCTGGAGGTCCT; NET mRNA forward ATTCTGCAGGGGCCGAGCCT and reverse ATCACAGGGAGCCGCTTGGC; TNFSF9 mRNA forward CAAAATGTTCTGCTGATCGATGG and reverse CGCCGCAGCTCTAGTTGAA; TP53 mRNA forward ACAAGCACCCTCAAGGGGGTCA and reverse TGCTGGCATTTGCACCTACCTCA; TUFM mRNA forward GCTGCCGCAAGATTAGGTT and reverse TGTTCTCCCTGACTTGGGAC; SCARNA4 RNA forward CAGTGAAGGCTGCTCTCTCC and reverse CTGGAGGACTAAGAAGGCTGA; SNORNA46 RNA forward CAACAGCAGAATGGCACAAG and reverse TTTAAACCTGTGGATGGGAAT; SNORD94 RNA forward GCTGAGTCATGGGAGCTGA and reverse AGGCTCAGATTGAGGCAACA; ACTB mRNA forward GCCCTGAGGCACTCTTCCA and reverse GCGGATGTCCACGTCACAC; Let-7i, UUUGUUCGUUCGGCUCGCGUGA.

POTS recruitment. Patients with POTS sharing the common clinical characteristics were recruited through our orthostatic intolerance clinic. Individuals with POTS who had participated in prior research studies were recruited for epigenetic analysis. All participants with POTS were taking medications including fludrocortisone, midodrine, propranolol, atenolol, pyridostigmine, topiramate, citalopram, thyroxine, amitriptyline, benztropine, and dihydroergotamine. Control participants were taking no medications other than the oral contraceptive pill in 3 participants and finasteride in 1 participant. Participants provided 50-100 ml of venous blood collected in EDTA-containing vacutainer tubes and leukocytes were isolated using Ficoll.

Ex vivo vorinostat experiments. Leukocytes isolated from healthy and POTS participants were incubated in DMEM with $10 \%$ FBS and antibiotic/antimycotic with TSA $(500 \mathrm{nM})$ or vorinostat $(2 \mu \mathrm{M})$ in a 6 -well plate. Approximately $3 \times 10^{6}$ cells were seeded in each well. After overnight incubation at $37^{\circ} \mathrm{C}$ and $5 \%$ $\mathrm{CO}_{2}$, cells were harvested and RNA was extracted for gene expression using qRT-PCR.

Statistics. Data are presented as mean \pm SEM or SD as indicated in each group. All data were evaluated with 1-tailed or 2-tailed, unpaired or paired (where appropriate) Student's $t$ test using QuickCals (Graphpad). A $P$ value less than or equal to 0.05 was considered statistically significant.

Study approval. This study was approved by Alfred Hospital Ethics Committee (approval number 268/10). There was no clinical trial listing associated with this study. Written specific genetics consent for this study was received from the participants prior to inclusion.

Supplemental data. Further information can be found in the Supplemental Data.

\section{Author contributions}

$\mathrm{AK}$ and AE designed the study. AK developed RICh and ChIP-RNase. AK and HK obtained leukocytes from human blood. MZ performed deep sequencing. AK, MZ, and HR analyzed sequencing data. JO and $\mathrm{AK}$ performed MeCP2 knockdown experiments. SC and ME recruited patients and collected blood. AE and AK prepared figures and cowrote the manuscript, which has been edited by AK, HR, MZ, ME, SM, and HK. All authors have read and approved the submitted manuscript. 


\section{Acknowledgments}

We would like to acknowledge Gautam Vaddadi and Elisabeth Lambert (Baker IDI Heart and Diabetes Institute) who performed the head-up tilt (HUT) studies. This study was supported by a National Health and Medical Research Council (NHMRC) program grant ME, research fellowship AE (1070386 and APP0526681), and NHMRC-NFSC joint program AE (APP1113188). This work was supported in part by the Victorian Government's Operational Infrastructure Support Program.

Address correspondence to: Assam El-Osta, Central Clinical School, Faculty of Medicine, Level 5, Epigenetics in Human Health and Disease Laboratory, 99 Commercial Road, Melbourne, Victoria 3004, Australia. Phone: 613.99030477; E-mail: sam.el-osta@monash.edu.

1. Shannon JR, et al. Orthostatic intolerance and tachycardia associated with norepinephrine-transporter deficiency. $N$ Engl $J$ Med. 2000;342(8):541-549.

2. Beane M, Marrocco RT. Norepinephrine and acetylcholine mediation of the components of reflexive attention: implications for attention deficit disorders. Prog Neurobiol. 2004;74(3):167-181.

3. Pliszka SR. The neuropsychopharmacology of attention-deficit/hyperactivity disorder. Biol Psychiatry. 2005;57(11):1385-1390

4. Schlaich MP, et al. Sympathetic augmentation in hypertension: role of nerve firing, norepinephrine reuptake, and angiotensin neuromodulation. Hypertension. 2004;43(2):169-175.

5. Goldstein DS, Brush JE, Eisenhofer G, Stull R, Esler M. In vivo measurement of neuronal uptake of norepinephrine in the human heart. Circulation. 1988;78(1):41-48.

6. Bayles R, et al. Epigenetic modification of the norepinephrine transporter gene in postural tachycardia syndrome. Arterioscler Thromb Vasc Biol. 2012;32(8):1910-1916.

7. Harikrishnan $\mathrm{KN}$, et al. Alleviating transcriptional inhibition of the norepinephrine slc6a2 transporter gene in depolarized neurons. J Neurosci. 2010;30(4):1494-1501

8. Harikrishnan $\mathrm{KN}$, et al. Brahma links the SWI/SNF chromatin-remodeling complex with MeCP2-dependent transcriptional silencing. Nat Genet. 2005;37(3):254-264.

9. Chahrour M, et al. MeCP2, a key contributor to neurological disease, activates and represses transcription. Science. 2008;320(5880):1224-1229.

10. Goldstein DS, et al. Cardiac sympathetic dysautonomia in chronic orthostatic intolerance syndromes. Circulation. 2002;106(18):2358-2365.

11. Bovia F, et al. Efficient transduction of primary human B lymphocytes and nondividing myeloma B cells with HIV-1-derived lentiviral vectors. Blood. 2003;101(5):1727-1733.

12. Young JI, et al. Regulation of RNA splicing by the methylation-dependent transcriptional repressor methyl-CpG binding protein 2. Proc Natl Acad Sci USA. 2005;102(49):17551-17558.

13. Dandrea M, Donadelli M, Costanzo C, Scarpa A, Palmieri M. MeCP2/H3meK9 are involved in IL-6 gene silencing in pancreatic adenocarcinoma cell lines. Nucleic Acids Res. 2009;37(20):6681-6690.

14. Jones PL, et al. Methylated DNA and MeCP2 recruit histone deacetylase to repress transcription. Nat Genet. 1998;19(2):187-191.

15. Waibel M, et al. Manipulation of B-cell responses with histone deacetylase inhibitors. Nat Commun. 2015;6:6838.

16. Roger T, et al. Histone deacetylase inhibitors impair innate immune responses to Toll-like receptor agonists and to infection. Blood. 2011;117(4):1205-1217.

17. Li D, Marchenko ND, Moll UM. SAHA shows preferential cytotoxicity in mutant p53 cancer cells by destabilizing mutant p53 through inhibition of the HDAC6-Hsp90 chaperone axis. Cell Death Differ. 2011;18(12):1904-1913.

18. Harms MP, van Lieshout JJ. Cerebrovascular and cardiovascular responses associated with orthostatic intolerance and tachycardia. Clin Auton Res. 2001;11(1):35-38.

19. Mitchell EA, Gentet LJ, Dempster J, Belelli D. GABAA and glycine receptor-mediated transmission in rat lamina II neurones: relevance to the analgesic actions of neuroactive steroids. J Physiol (Lond). 2007;583(Pt 3):1021-1040.

20. Cifuentes-Rojas C, Hernandez AJ, Sarma K, Lee JT. Regulatory interactions between RNA and polycomb repressive complex 2 . Mol Cell. 2014;55(2):171-185.

21. Razin A. CpG methylation, chromatin structure and gene silencing-a three-way connection. EMBO J. 1998;17(17):4905-4908

22. Bayles R, Baker E, Eikelis N, El-Osta A, Lambert G. Histone modifications regulate the norepinephrine transporter gene. Cell Cycle. 2010;9(22):4600-4601.

23. Nan X, Campoy FJ, Bird A. MeCP2 is a transcriptional repressor with abundant binding sites in genomic chromatin. Cell. 1997;88(4):471-481.

24. Yasui $\mathrm{DH}$, et al. Integrated epigenomic analyses of neuronal MeCP2 reveal a role for long-range interaction with active genes Proc Natl Acad Sci USA. 2007;104(49):19416-19421.

25. Galvão TC, Thomas JO. Structure-specific binding of MeCP2 to four-way junction DNA through its methyl CpG-binding domain. Nucleic Acids Res. 2005;33(20):6603-6609.

26. Cukier HN, Perez AM, Collins AL, Zhou Z, Zoghbi HY, Botas J. Genetic modifiers of MeCP2 function in Drosophila. PLoS Genet. 2008;4(9):e1000179.

27. Zhu W, et al. MicroRNA expression analysis: clinical advantage of propranolol reveals key microRNAs in myocardial infarction. PLoS ONE. 2011;6(2):e14736

28. Jeffery L, Nakielny S. Components of the DNA methylation system of chromatin control are RNA-binding proteins. J Biol Chem. 2004;279(47):49479-49487. 
29. Maxwell SS, Pelka GJ, Tam PP, El-Osta A. Chromatin context and ncRNA highlight targets of MeCP2 in brain. RNA Biol. 2013;10(11):1741-1757.

30. Udali S, Guarini P, Moruzzi S, Choi SW, Friso S. Cardiovascular epigenetics: from DNA methylation to microRNAs. Mol Aspects Med. 2013;34(4):883-901.

31. Puthanveetil P, Chen S, Feng B, Gautam A, Chakrabarti S. Long non-coding RNA MALAT1 regulates hyperglycaemia induced inflammatory process in the endothelial cells. J Cell Mol Med. 2015;19(6):1418-1425.

32. Shan K, et al. Role of long non-coding RNA-RNCR3 in atherosclerosis-related vascular dysfunction. Cell Death Dis. 2016;7(6):e2248.

33. Long SW, Ooi JY, Yau PM, Jones PL. A brain-derived MeCP2 complex supports a role for MeCP2 in RNA processing. Biosci Rep. 2011;31(5):333-343.

34. Rafehi H, et al. Vascular histone deacetylation by pharmacological HDAC inhibition. Genome Res. 2014;24(8):1271-1284

35. Kong Y, et al. Suppression of class I and II histone deacetylases blunts pressure-overload cardiac hypertrophy. Circulation. 2006;113(22):2579-2588.

36. Zhao TC, Cheng G, Zhang LX, Tseng YT, Padbury JF. Inhibition of histone deacetylases triggers pharmacologic preconditioning effects against myocardial ischemic injury. Cardiovasc Res. 2007;76(3):473-481.

37. Colussi C, et al. The histone deacetylase inhibitor suberoylanilide hydroxamic acid reduces cardiac arrhythmias in dystrophic mice. Cardiovasc Res. 2010;87(1):73-82

38. Xie M, et al. Histone deacetylase inhibition blunts ischemia/reperfusion injury by inducing cardiomyocyte autophagy. Circulation. 2014;129(10):1139-1151.

39. Keating ST, Plutzky J, El-Osta A. Epigenetic changes in diabetes and cardiovascular risk. Circ Res. 2016;118(11):1706-1722.

40. Rafehi H, El-Osta A. HDAC inhibition in vascular endothelial cells regulates the expression of ncRNAs. Non-Coding RNA. 2016;2(2):4

41. ENCODE Project Consortium. An integrated encyclopedia of DNA elements in the human genome. Nature. 2012;489(7414):57-74

42. Dobin A, et al. STAR: ultrafast universal RNA-seq aligner. Bioinformatics. 2013;29(1):15-21.

43. Yates A, et al. Ensembl 2016. Nucleic Acids Res. 2016;44(D1):D710-D716.

44. Liao Y, Smyth GK, Shi W. featureCounts: an efficient general purpose program for assigning sequence reads to genomic features. Bioinformatics. 2014;30(7):923-930

45. Robinson MD, McCarthy DJ, Smyth GK. edgeR: a Bioconductor package for differential expression analysis of digital gene expression data. Bioinformatics. 2010;26(1):139-140 